\title{
Le Li* and Li-yong Wang \\ Simulation of Dynamic Recrystallization Behavior under Hot Isothermal Compressions for as-extruded 3Cr20Ni10W2 Heat-Resistant Alloy by Cellular Automaton Model
}

https://doi.org/10.1515/htmp-2017-0025

Received March 3, 2017; accepted June 28, 2017

\begin{abstract}
In order to study dynamic recrystallization behavior of the as-extruded 3Cr20Ni10W2 under isothermal compression conditions, a cellular automaton (CA) model was applied to simulate hot compression. Analysis on the strainstress curves indicates that dynamic recrystallization is the main softening mechanism for the 3Cr20Ni10W2 when the deformation occurred in the temperature range of 1203-1303 $\mathrm{K}$ with an interval of $50 \mathrm{~K}$ and strain rate range of $0.01-10 \mathrm{~s}^{-1}$. The deformation temperature and strain rate have a significant influence on the dynamically recrystallized grain size. Subsequently, a CA model is established to simulate the dynamic recrystallization behaviors of the studied alloy. The simulated results show that the mean grain size increases with the increased deformation temperature and decreases with the increased strain rate, which is consistent with the experimental result. In addition, the average absolute relative error, which is $13.14 \%$, indicates that the process of the dynamic recrystallization and the dynamically recrystallized grain size can be well predicted by the present CA model.
\end{abstract}

Keywords: 3Cr20Ni10W2, cellular automaton, dynamic recrystallization, recrystallized grain size

\section{Introduction}

3Cr20Ni10W2, as a kind of heat-resistant alloys, has excellent heat-resistance and high corrosion resistance, high strength, and superior creep strength. For this reason, 3Cr20Ni10W2 is primarily used in manufacture of turbines components and exhaust valves on diesel engine [1, 2]. It is

\footnotetext{
*Corresponding author: Le Li, The Ministry of Education Key Laboratory of Modern Measurement and Control Technology, Beijing Information Science and Technology University, Beijing 100192, China, E-mail: lilelile2004@21cn.com

Li-yong Wang, The Ministry of Education Key Laboratory of Modern Measurement and Control Technology, Beijing Information Science and Technology University, Beijing 100192, China; Collaborative Innovation Center of Electric Vehicles in Beijing, Beijing Information Science and Technology University, Beijing 100192, China, E-mail: wangliyong2004@163.com
}

well known that mechanical properties of the metal are mainly decided by microstructure such as grain size to a larger extent. Usually, the three typical metallurgical phenomena during the hot deformation, namely work hardening (WH), dynamic recovery (DRV), and dynamic recrystallization (DRX), has a decisive influence on grain size, of which DRX is generally recognized a phenomenon that new high-angle, mobile grain boundaries generated and migrated, in other words, DRX as a kind of mechanism of grain refinement has dominant influence on the final grain size. However, it is tedious to observe the microstructural evolution process during grain growth by physical experiments. Therefore, more and more attention is paid to develop an efficient method to simulate the grain size evolution when the hot deformation occurred to the metal.

In recent years, two approaches are commonly supposed to simulate the DRX with the development of computer simulation technology, i.e. the Monte Carlo (MC) method and the cellular automaton (CA) method [3]. For instance, MC method was used to study microstructural evolution such as static recrystallization, DRX and grain growth [4-6]. However, grain growth kinetics during DRX was not simulated by this method. Subsequently, CA model gets noticed gradually, which provides an approach describing the growth kinetics and microstructure evolution during the hot deformation process [7]. In other words, the CA model can describe the DRX and the grain growth graphically. Specifically, the CA is a mathematical method which is generally adopted to reveal the complex system evolution rules in the discrete space-time by an application of deterministic or probabilistic transformation rules to the location of a lattice. Every cell is not isolated, and its present state and next state are decided by the neighbor cells based on the above mathematical arithmetic $[8,9]$ Meanwhile dislocation density in the CA algorithm acted as a very significant role in nucleation and microstructural evolution of DRX during hot deformation, and then the modified LJ model, which has been proved that it is suitable to simulate the dislocation density evolution in detail [10], recommended by GOURDET and MONTHEULLET [11] was adopted to describe the dislocation densities evolution. For example, Chen et al. [12] presented a CA model to predict 
the DRX behaviors of 30Cr2Ni4MoV rotor steel during hot deformation. In order to overcome the difficulty inaccurately determining the DRX nucleation parameters for low carbon steel, Jin and Cui [13] developed a flow stress-based inverse analysis method, which was implemented by coupling a CA model with an adaptive response surface method. In addition, E. Afshari et al. studied the kinetics of static recrystallization and distribution of recrystallized grain size by means of a cellular automata model together with the stored energy calculated by the deformation analysis [14]. Yan-Xing Liu et al. [15] Study of DRX in a Ni-based superalloy by experiments and CA model.

In present paper, DRX model and the model for flow stress of as-extruded 3Cr20Ni10W2 were established based on the true stress-strain data, and then the various parameters in the dislocation density model, recovery model and nucleation and growth of DRX were determined respectively. Subsequently, the grain size evolution under different deformation conditions was modeled through the present CA model.

\section{Experimental procedure}

The specific experimental has already been reported [16]. Briefly, the chemical compositions of 3Cr20Ni10W2 heat- resisting alloy are (wt.\%) C 0.25, Si 1, Cr 20, Ni 10, Mn 1, W 2, Fe (balance). The experimental samples with same diameter of $10 \mathrm{~mm}$ and height of $12 \mathrm{~mm}$ were subjected to the designed experimental steps, detailed, the samples were heated to a specified temperature at a heating rate of $1 \mathrm{~K} / \mathrm{s}$ by the Gleeble 1500 machine, and held at the specified temperature for $180 \mathrm{~s}$ to ensure a uniform starting temperature and decrease the material anisotropy. All the twenty specimens were compressed to a fixed true strain of 0.9163 at four different temperatures of $1203 \mathrm{~K}$, $1253 \mathrm{~K}, 1303 \mathrm{~K} 1353 \mathrm{~K}$ and $1403 \mathrm{~K}$, and four different strain rates of $0.01 \mathrm{~s}^{-1}, 0.1 \mathrm{~s}^{-1}, 1 \mathrm{~s}^{-1}$ and $10 \mathrm{~s}^{-1}$ and then a series of strain-stress data was collected. Subsequently, the microstructures on the section plane of specimen deformed to the true strain of -0.91 were examined and analyzed under the optical microscope. By means of the metallography, the grain size under different deformation conditions was counted [17].

\section{Analysis of strain-stress curve flow behavior}

As shown in Figure 1 (a d), the true compressive stressstrain curves of 3Cr20Ni10W2 heat-resistant alloy

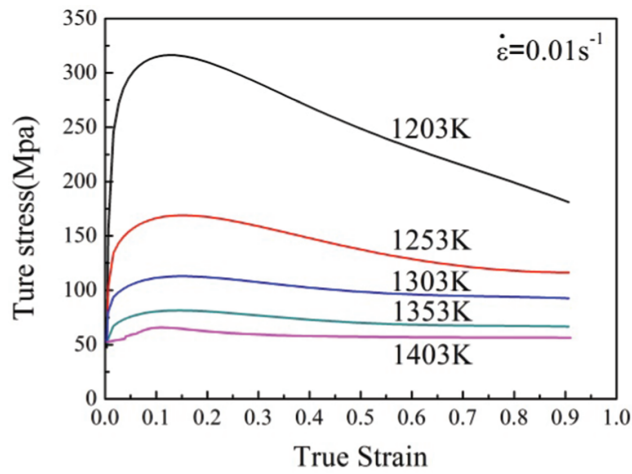

(a)

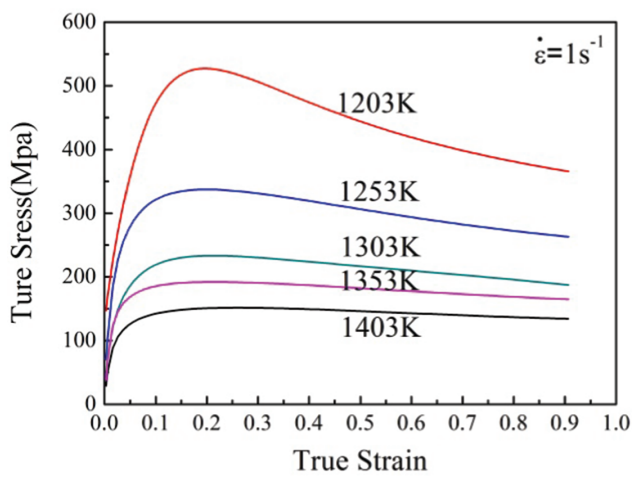

(c)

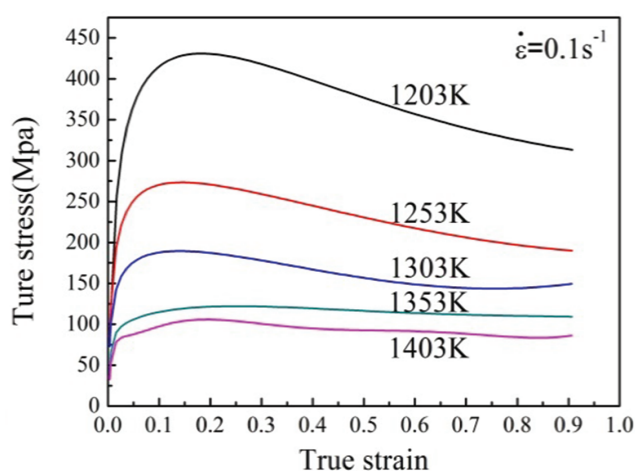

(b)

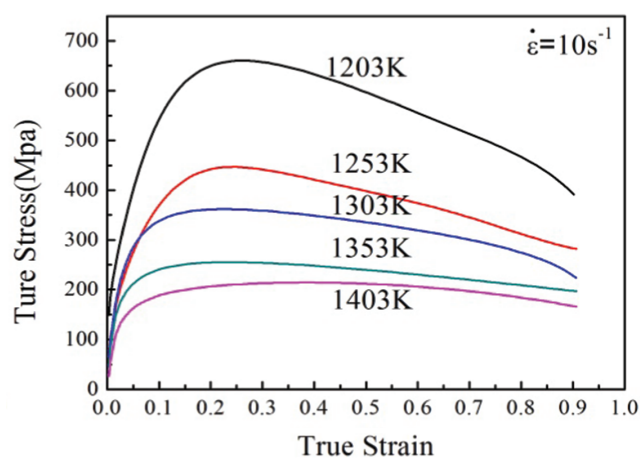

(d)
Figure 1: True stress-strain curves of 3 Cr20Ni10W2 alloy at different strain rates and temperatures (a) $0.01 \mathrm{~s}^{-1}$, $1203 \sim 1403 \mathrm{~K}$; (b) $0.1 \mathrm{~s}^{-1}$, 1203 1403 K; (c) $1 \mathrm{~s}^{-1}$, $1203 \sim 1403 \mathrm{~K}$; (d) $10 \mathrm{~s}^{-1}$, $1203 \sim 1403 \mathrm{~K}$. 
described the stress change with the variation of strain under the different strain rate and temperature. After analysis of these curves in whole, it can be found that, for all of specimens, the flow stress decreases monotonically with different softening rates. Specifically, for a fixed strain rate, the flow stress decreases obviously with the increased temperature, and the flow stress increases with the increased strain rate at a fixed temperature.

In addition, the previous study [16] divided the stress evolution into three distinct stages including WH, DRX, and DRV, and the detail type classification is concluded as following: decreasing gradually to a steady state with DRV softening $\left(1403 \mathrm{~K} \& 0.01 \mathrm{~s}^{-1}, 1353 \sim 1403 \mathrm{~K} \& 0.1 \mathrm{~s}^{-1}, 1403 \mathrm{~K}\right.$ $\& 1 \mathrm{~s}^{-1}$ ), and decreasing continuously with significant DRX softening (1203 1303 K \& $0.01 \mathrm{~s}^{-1}$, 1203 1303 K \& $0.1 \mathrm{~s}^{-1}$, 1203 1353 K \& $1 \mathrm{~s}^{-1}$, 1203 1403 K \& $10 \mathrm{~s}^{-1}$ ).

\section{The establishment of a cellular automata model (CA)}

CA model is gradually utilized in studying microstructure evolution $[15,18,19]$. In this method, the discrete lattice geometry is square. Subsequently, the simulated area was defined, the number of rows and columns of discrete lattice points in the microstructure was set as $200 \times 200$ and every cell in the discrete lattice is $2 \mathrm{um}$, thus this would result in a microstructure simulation of a region of material $400 \times 400 \mathrm{um}$. The boundary conditions is periodic wraparound by default, then the neighbor type was set as the Moore `s, in addition, the radius of the neighbor is 1, thus, the adjacent cell is 8 . For an annealed metal, the dislocation density was typically taken as 0.01 . The dislocation density for each region of microstructure is calculated as a function of hardening and recovery with respect to strain. The type of nucleation conditions for new grains was threshold dislocation density and probability. In the end, the initial grain size was input and the value is $63.9 \mathrm{um}$.

\section{Dislocation density model}

According to Yoshie-Lassraoui-Jonas (YLJ) model [20], dislocation density is defined as a function of recovery and hardening coefficients, and the expression is shown as following:

$$
d \rho_{i}=\left(h-r \rho_{i}\right) d \varepsilon
$$

The effect of WH and the DRV on the evolution of dislocation density during the plastic deformation $(d \rho / d \varepsilon)$ may be described by the difference between WH term (h) and the DRV term $(\mathrm{r} \rho)$ [21]. In addition, WH coefficients $(h)$ and recovery coefficients $(r)$ are described as function of strain rate and temperature, the two expressions are shown as following:

$$
\begin{aligned}
& h=h_{0}\left(\frac{\dot{\varepsilon}}{\dot{\varepsilon}_{0}}\right)^{-m} \exp \left(\frac{m Q}{R T}\right) \\
& r=r_{0}\left(\frac{\dot{\varepsilon}}{\dot{\varepsilon}_{0}}\right)^{-m} \exp \left(\frac{m Q}{R T}\right)
\end{aligned}
$$

where $m$ is the hardening sensitivity of deformation here the value of $m$ is $0.2 ; Q$ is the apparent activation energy; $h_{0}$ is the hardening constant; $r_{0}$ is the recovery constant; $\dot{\varepsilon}_{0}$ is the strain rate calibration constant, which is usually assigned a value of 1 . The relationship between stress and dislocation density may be expressed by the following function [22].

$$
\sigma=M \alpha_{1} G b \sqrt{\rho}
$$

where $M$ is the Taylor factor; Integration of eq. (1), and substitution of eq. (4) obtains:

$$
\sigma=\left[\sigma_{\infty}^{2}-\left(\sigma_{\infty}^{2}-\sigma_{e}^{2}\right) \exp (-r \varepsilon)\right]^{1 / 2}
$$

After solving the first derivatives of the $\sigma$ versus $\varepsilon$ and $\rho$ versus $\varepsilon$, the eq. (4) was transferred to the below expression.

$$
\frac{d \rho}{d \varepsilon}=\frac{1}{\left(M \alpha_{1} G b\right)^{2}} 2 \sigma \frac{d \sigma}{d \varepsilon}
$$

Combined the eqs (1), (4) and eqs (6), (7) was deduced.

$$
2 \sigma \frac{d \sigma}{d \varepsilon}=r \sigma_{\infty}^{2}-r \sigma^{2}
$$

The slope of the plot of $2 \sigma \frac{d \sigma}{d \varepsilon}$ versus $\sigma^{2}$ is the $r$ and then the intersection with the vertical axis is used to confirm the $h$ by eq. (4).

\section{Recovery model}

DRV is a kind of softening mechanics by which metals and alloys undergo a reduction in the density of accumulated dislocations during plastic deformation at elevated temperatures. The common recovery model adopted in the CA model is established by Goetz and Seetharaman [23], which is sampling a certain number of cells $(N)$ and reduced the dislocation density by half in next time step. The expressions are shown as following: 


$$
\begin{gathered}
\rho_{i, j}^{t}=\rho_{i, j}^{t} / 2 \\
N=\left(\frac{\sqrt{2} M}{K}\right)^{2} \cdot \dot{\rho}^{2}
\end{gathered}
$$

where $M$ is the total number of cells in the CA model; $K$ is a constant which usually take a value of $6030 ; \dot{\rho}$ is the dislocation multiplication rate.

\section{DRX nucleation and growth model}

For the DRX nucleation, it is accepted that the dislocation density plays a controlled role in the nucleation of dynamically recrystallized grain. Specifically, once the dislocation density exceeds a critical level, the nucleation of DRX starts immediately. In the present CA model, the assumption that nucleation appeared at a constant rate is adopted [24], and here a function of deformation temperature and strain rate is adopted to describe the constant rate of nucleation of one unit of grain boundary area [25].

$$
\dot{n}(\dot{\varepsilon}, T)=C \dot{\varepsilon}^{m} \exp \left(-\frac{Q_{a c t}}{R T}\right)
$$

where $C$ and $m$ are material constants, and the value of $m$ is 1 , the value of $C$ is $200 . \dot{\varepsilon}$ is the strain rate, $Q_{a c t}$ is the activation energy, $R$ is the universal gas constant and its value is taken 8.31 , and $T$ is the absolute temperature (K).

DRX grain appears when the dislocation density reaches the critical value, new grains firstly appears nearby older grain boundary at the beginning of the DRX, the initial strain and dislocation density of new grains is considered as 0 . Obviously, a difference of dislocation density between recrystallization grain and older grain exists, which gives driving force to grain growth. Afterwards, new grains start to grow up until the driving force decreases to 0 , by this time the new grains contacted to each other and no longer grow up. Similarly, the dislocation density of the new grains increases with the increase of deformation, which is responsible for the nucleation and growth of DRX again. Thus, based on the change of energy, the driving force for the growth of new grains can be deduced. Generally, the energy change is composed of two parts including volume energy change and surface energy change [26].

$$
d E=d E_{\text {vol }}+d E_{\text {surf }}=\frac{4}{3} \pi r^{3} \tau \Delta \rho+4 \pi r^{2} \gamma
$$

In the eq. (11), the grain is assumed as the sphere, and the expression of driving force is the first derivative of $E$ with respect to $r$, the expression is shown below:

$$
F=-\frac{d E}{d r}=4 \pi r^{2} \tau \Delta \rho-8 \pi r \gamma
$$

where $r$ is the dynamically recrystallized grain radius, $\tau$ is energy of the dislocation line, $\Delta \rho$ is the dislocation density difference between the growing grains and its adjacent grains. $\gamma$ is the grain boundary energy, and the function on $\gamma$ is expressed below [27].

$$
\gamma=\left\{\begin{array}{cc}
\gamma_{m} & \theta \geq \theta_{c} \\
\gamma_{m} \frac{\theta_{i}}{\theta_{c}}\left(1-\ln \left(\frac{\theta_{i}}{\theta_{c}}\right)\right) & \theta<\theta_{c}
\end{array}\right.
$$

where $\theta$ is the orientation difference between two neighboring grains. $\gamma_{m}$ is the energy of high angle boundary, $\theta_{c}$ is the critical orientation difference for high angle grain boundary, which is usually accepted as a value of $15^{\circ} \cdot \gamma_{m}$ can be calculated as the following function.

$$
\gamma_{m}=\frac{\mu b \theta_{m}}{4 \pi(1-v)}
$$

where the $v$ is Poisson ratio.

\section{DRX volume fraction model and grain size model}

\section{Initiation of DRX}

As mentioned above, twelve deformation conditions with distinct DRX characteristic corresponding to three temperatures of $1203 \mathrm{~K}, 1253 \mathrm{~K}$ and $1303 \mathrm{~K}$, and four strain rates of $0.01 \mathrm{~s}^{-1}, 0.1 \mathrm{~s}^{-1}, \mathrm{~s}^{-1}$ and $10 \mathrm{~s}^{-1}$ were selected, Based on the strain-stress data of the twelve curves, the strain hardening rate $d \sigma / d \varepsilon$ versus $\sigma$ curves were plotted as shown in Figure 2, it is recognized that the critical conditions for DRX can be determined when the minimum value of $|-d \theta / d \sigma|$ appeared, where the $\theta$ was defined as $\theta=d \sigma / d \varepsilon$ [28]. According to this method, the critical strains $\left(\varepsilon_{c}\right)$ and critical stresses under these twelve deformation conditions were calculated, and the linear relationship between $\varepsilon_{c}$ and the strain for the peak stress $\left(\varepsilon_{p}\right)$ is summarized as the eq. (15) [29]. As for the $\varepsilon_{p}$, it is usually recognized that the value of $\varepsilon_{p}$ is controlled by strain rate $(\dot{\varepsilon})$, deformation activation energy $\left(Q_{1}\right)$, the initial grain size $\left(d_{0}\right)$, and deformation temperature $(T)$, and this relationship is expressed as eq. (15) [30, 31].

$$
\varepsilon_{c}=\alpha \varepsilon_{p}
$$




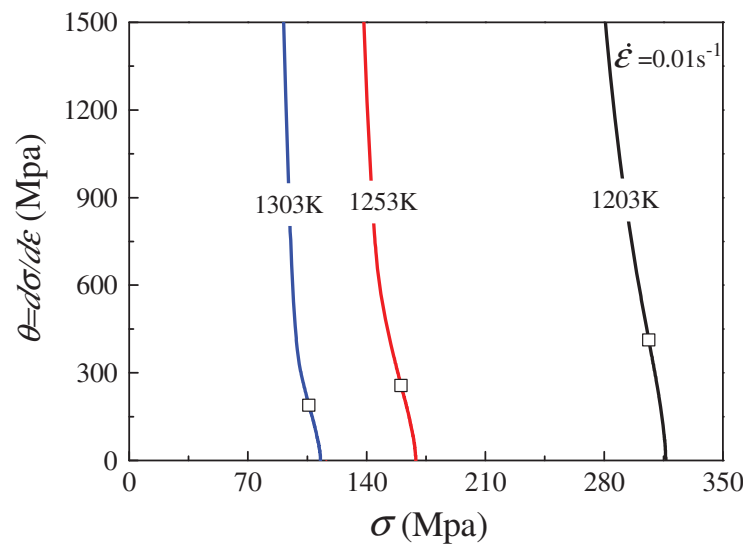

(a)

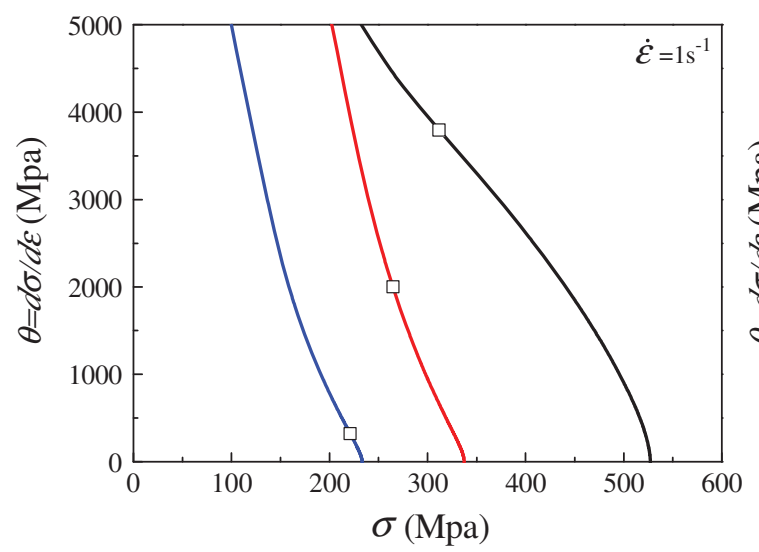

(c)

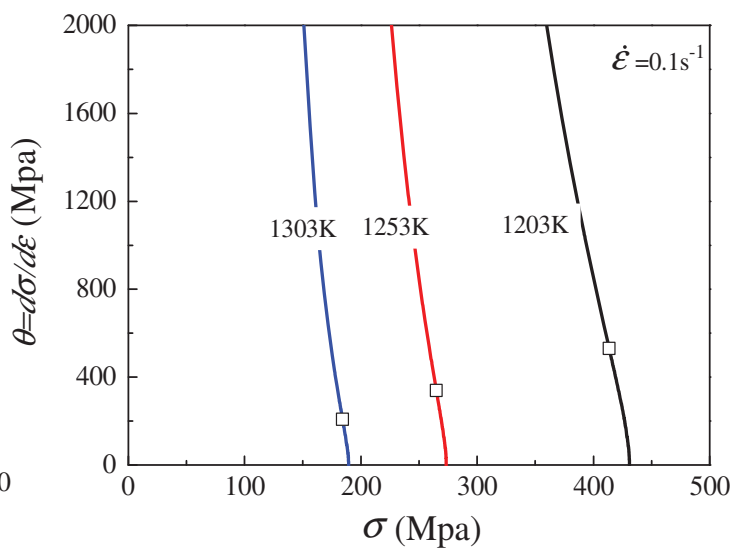

(b)

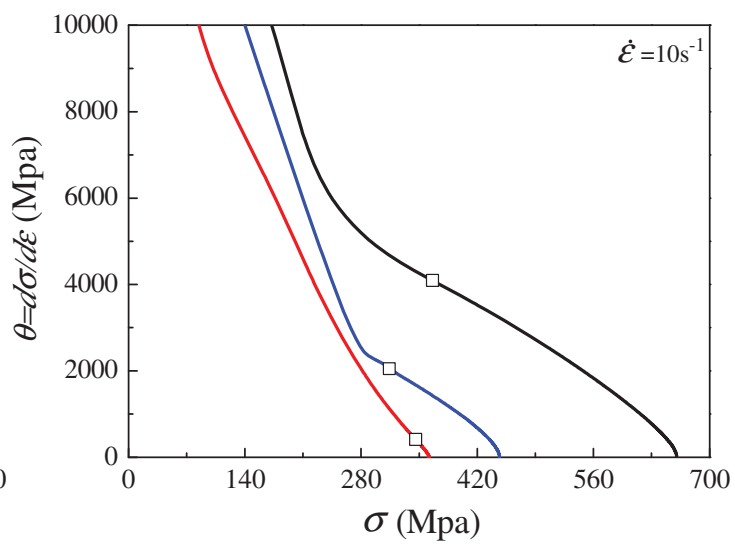

(d)

Figure 2: $d \sigma / d \varepsilon$ versus $\sigma$ plots up to the peak points of the true stress-strain curves at different deformation temperatures and strain rates of: (a) $0.01 \mathrm{~s}^{-1}$, (b) $0.1 \mathrm{~s}^{-1}$, (c) $1 \mathrm{~s}^{-1}$, (d) $10 \mathrm{~s}^{-1}$.

where $\alpha$ is a material constant.

$$
\varepsilon_{p}=\alpha_{1} d_{0}{ }^{n_{1}} \dot{\varepsilon}^{m_{1}} \exp \left(Q_{1} / R T\right)
$$

where $a_{1}, n_{1}$ and $m_{1}$ are material constants, $R$ is the universal gas constant $\left(8.31 \mathrm{~J} \mathrm{~mol}^{-1} \mathrm{~K}^{-1}\right)$. In this work, the effect of initial grain size on the peak strain was without consideration, so eq. (16) can be described to eq. (17).

$$
\varepsilon_{p}=\alpha_{1} \dot{\varepsilon}^{m_{1}} \exp \left(Q_{1} / R T\right)
$$

(1) Calculation of material constant $a$

As shown in Figure 3, in the basis of the values of $\varepsilon_{c}$ and $\varepsilon_{p}$ under different temperatures and strain rates, the relationships between $\varepsilon_{c}$ and $\varepsilon_{p}$ were fitted linearly. The slope value of this line is the value of $a$, and its value was 0.1069 .

(2) Determination of $m_{1}, Q_{1}$ and $a_{1}$

Take natural logarithms on both sides of eq. (17), the eq. (18) is obtained.

$$
\ln \varepsilon_{p}=\ln \alpha_{1}+m_{1} \ln \dot{\varepsilon}+Q / R T
$$

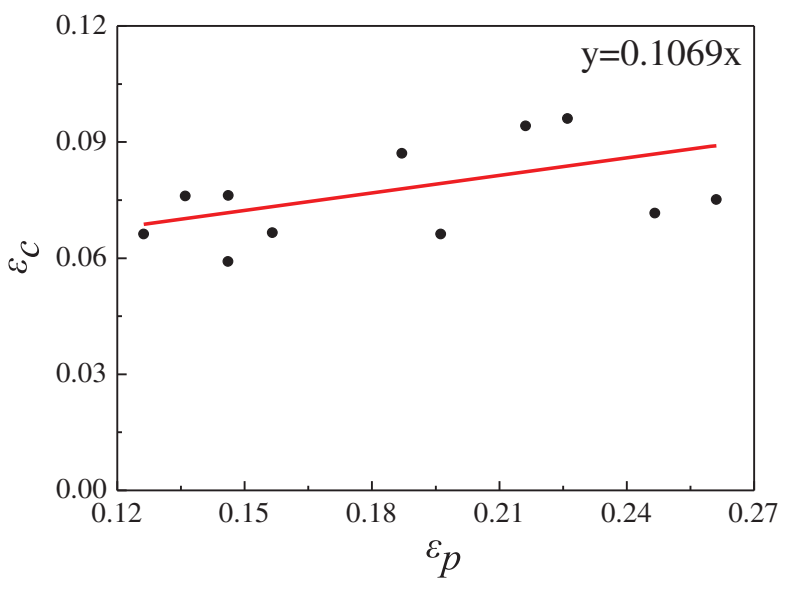

Figure 3: The relationships between $\varepsilon_{c}$ and $\varepsilon_{p}$.

Obviously, $m_{1}=\partial \ln \varepsilon_{p} / \partial \ln \dot{\varepsilon}$. As shown in Figure 4, the relationships between $\ln \varepsilon_{p}$ and $\ln \dot{\varepsilon}$ under different temperatures were fitted linearly. Every slope value of fitted line under a certain temperature and strain rates is the value of $m_{1}$. Observed from Figure 4, the slope values of the three 


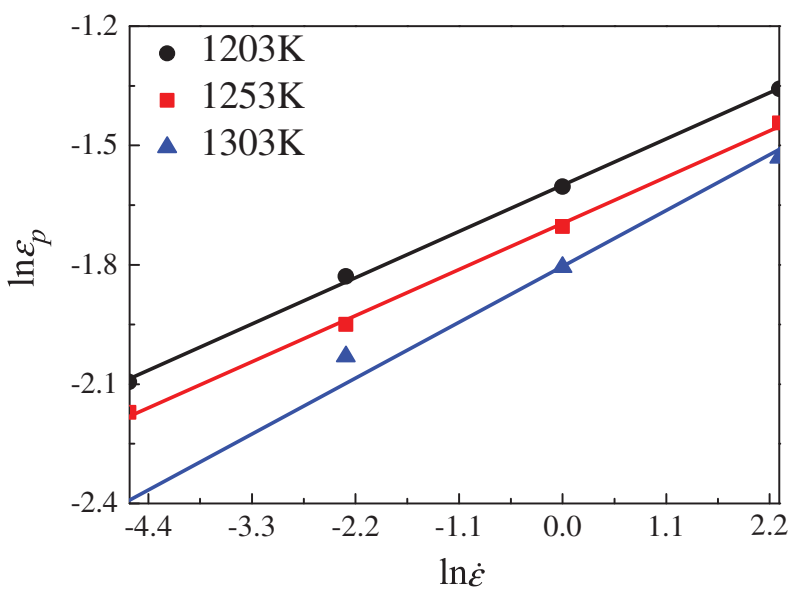

Figure 4: The relationships between $\partial \ln \varepsilon_{p}$ and $\partial \ln \dot{\varepsilon}$.

fitted lines under different strain rates were nearly equal. Therefore, the mean value of all the three slope values is recognized as the value of $m_{1}$, and value of $m_{1}$ was 0.113 .

From eq. (17), it is noticed that $Q_{1}=R \partial \ln \varepsilon_{p} / \partial(1 / T)$. As shown in Figure 5, the linear relationships under different strain rates between $\ln \varepsilon_{p}$ and $1 / T$ were described. The slope value under different strain rates was value of $\partial \ln \varepsilon_{p} / \partial(1 / T)$. It is observed that the four slopes were almost equal. Therefore the value of material constant $Q_{1}$ is equal to the mean value of the four slopes, and its value is $32251.664 \mathrm{~J} \mathrm{~mol}^{-1}$. Afterwards, $m_{1}$ and $Q_{1}$ were substituted into eq. (18) to calculate twelve values of material constant $a_{1}$ under the deformation conditions above. Finally $a_{1}$ was calculated as 0.0082 .

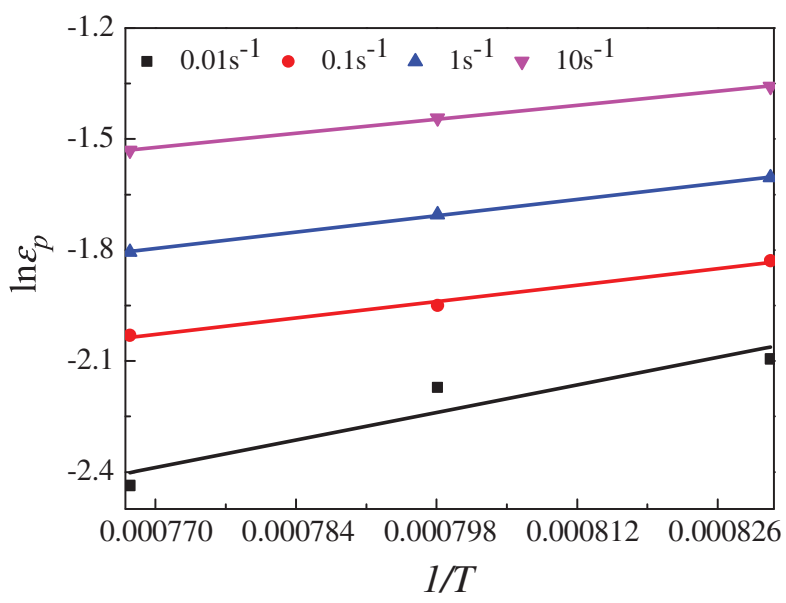

Figure 5: The relationships between $\partial \ln \varepsilon_{p}$ and $1 / \mathrm{T}$.

\section{DRX kinetic model}

It is well known that nucleation and growth of DRX started when the dislocations increased and accumulated to a critical degree. Generally, the well-known Johnson-
Mehl-Avrami-Kolmogorov (JMAK) equation as shown eq. (19) is widely used to describe the relationships between DRX fraction and true strain [28-31].

$$
X_{D R X}=1-\exp \left[-\beta_{d}\left(\frac{\varepsilon-\varepsilon_{c}}{\varepsilon_{0.5}}\right)^{k_{d}}\right]
$$

where $X_{D R X}$ is the volume fraction of DRX grains, $\varepsilon$ is the true strain. $\varepsilon_{0.5}$ is the strain when $D R X$ volume fraction reached $50 \%, \beta_{d}$ and $k_{d}$ are the Avrami material constants. In addition, an empirical model as shown in the below $[29,31]$ was introduced to calculate the value of $\varepsilon_{0.5}$.

$$
\varepsilon_{0.5}=\alpha_{2} d_{0}^{n_{2}} \dot{\varepsilon}^{m_{2}} \exp \left(Q_{2} / R T\right)
$$

where $Q_{2}$ is the activation energy for recrystallization, and $\alpha_{2}, n_{2}$ and $m_{2}$ are all material constants.

Although DRX can be observed from the metallography, it is tedious to carry out extensive and quantitative metallography measurements, which results in a hard work to calculate of DRX volume fraction under different deformation conditions according to microstructures. However, flow stress of metal during hot deformation is an actual and indirect reflection of microstructure evolution, therefore, based on the further analysis of the true stress-strain data $X_{D R X}$ can be determined. In this work, eq. (21) $[32,33]$ shown in the below is used to calculate the $X_{D R X}$.

$$
D_{D R X}=\frac{\left(\sigma_{d r v x}\right)^{2}-\left(\sigma_{d r x x}\right)^{2}}{\left(\sigma_{d r v s s}\right)^{2}-\left(\sigma_{d r x s s}\right)^{2}}
$$

where $\sigma_{d r v s s}$ is final steady state stress, and $\sigma_{d v v x}$ is the and the transient state stress on the true stress-strain curve of ideal DRV (as shown in Figure 6); $\sigma_{d r x s s}$ is the

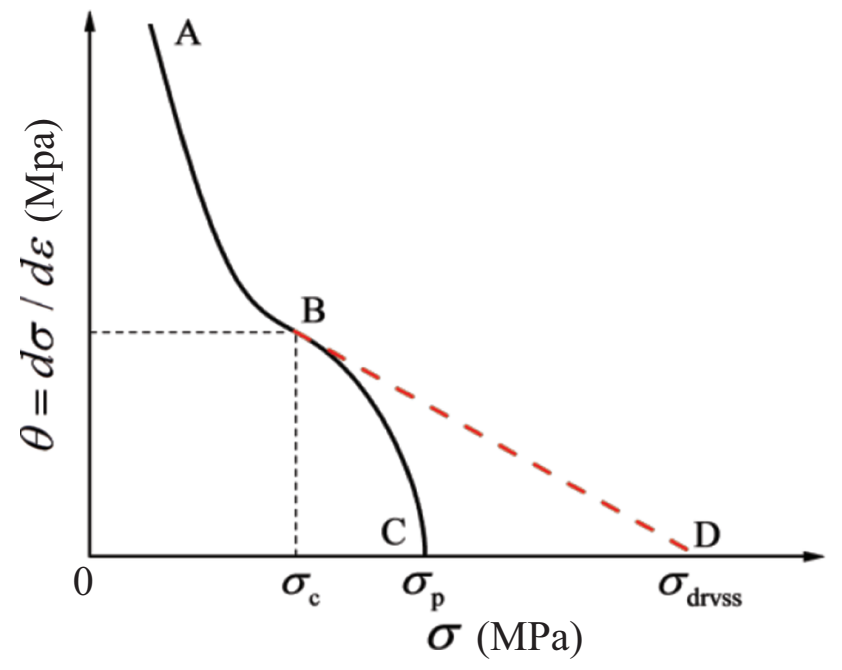

Figure 6: Plot of $\theta-\sigma$ employed to determine $\sigma_{d r v s s}$ by the intercept with the horizontal axis. 
final steady state stress, and $\sigma_{d r x x}$ is the transient state stress on actual DRX true stress-strain curve (as shown in Figure 6) plotted from a series of isothermal compression test data.

The method to determine the value of $\sigma_{d r v s}$ is shown below. Firstly, the critical stress $\left(\sigma_{c}\right)$ corresponding to the $\varepsilon_{c}$ can be obtained, and the $\varepsilon_{c}$ has been already determined above. Thus the $\theta$ corresponding to $\sigma_{c}$ was determined, based on the physical meaning, the $\sigma_{d r v s}$ is defined as a value, which is the value corresponding to point $\mathrm{D}$, in addition, it is obvious that the working-hardening behavior prior to $\sigma_{c}$ characterizes $\sigma_{d r v s}$ [34]. For the values of $\sigma_{d r v x}, \sigma_{d r x x}, \sigma_{d r v s s}$ and $\sigma_{d r x s s}$, which can be determined by $d \sigma / d \varepsilon$ versus $\sigma$ curves and the true stressstrain curves under different deformation conditions. Thus, the $X_{D R X}$ and the $\varepsilon_{0.5}$ can be calculated. Figure 7 describes the predicted DRX volume fractions under the different deformation conditions. Observed from Figure 7, it can be concluded that the deformation strain required for the same amount of DRX volume fraction decreases with the increased deformation temperature under a condition of fixed strain rate, which means that a low temperature is a poor condition for DRX, meanwhile, the deformation strain corresponding to the same amount of DRX volume fraction decreases with the decreased strain rate under a fixed temperature. This effect can be attributed to increased mobility of grain boundaries (growth kinetics) with the decreased strain rate and the increased temperature.

(1) Calculation of material constants: $\beta_{d}, k_{d}$

Taking natural logarithm on both sides of eq. (19) gives eq. (22).

$$
\ln \left[-\ln \left(1-X_{D R X}\right)\right] \varepsilon_{0.5}=\ln \beta_{d}+k_{d} \ln \left[\left(\varepsilon-\varepsilon_{c}\right) / \varepsilon_{0.5}\right]
$$

Therefore, $k_{d}=\partial \ln \left[-\ln \left(1-X_{D R X}\right)\right] / \partial \ln \left[\left(\varepsilon-\varepsilon_{c}\right) / \varepsilon_{0.5}\right]$. Take the values of the parameters into the $\ln \left[-\ln \left(1-X_{D R X}\right)\right]$ and $\ln \left[\left(\varepsilon-\varepsilon_{c}\right) / \varepsilon_{0.5}\right]$, then a series first derivatives values

$\mathrm{S}^{-1}$.

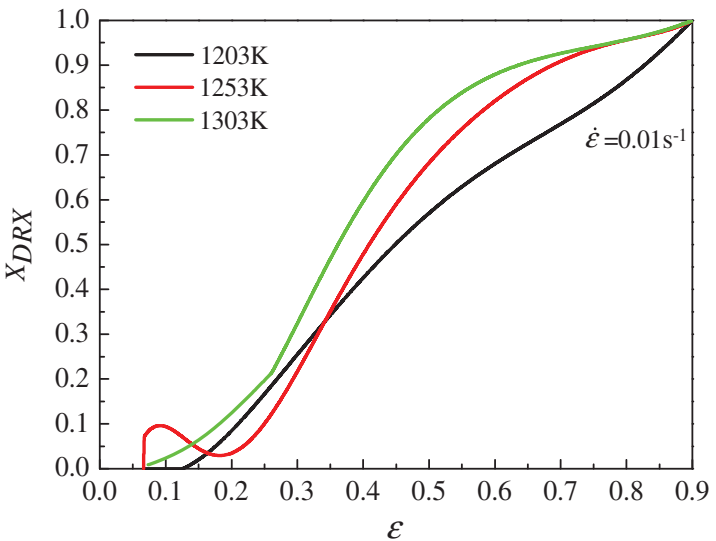

(a)

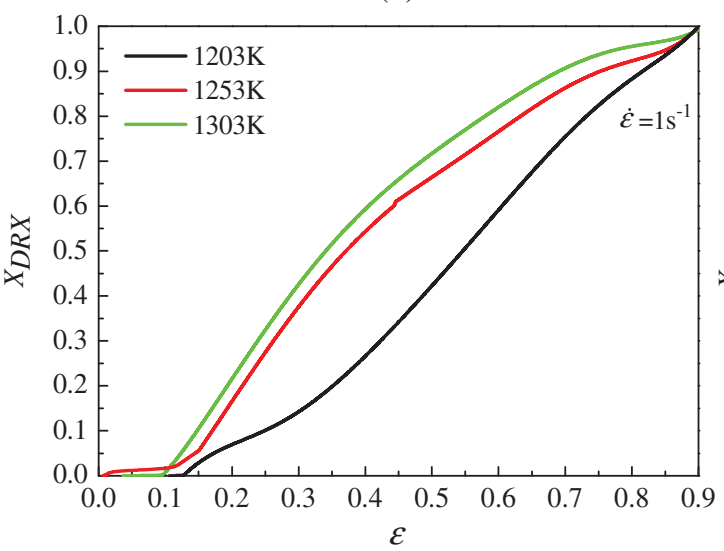

(c)

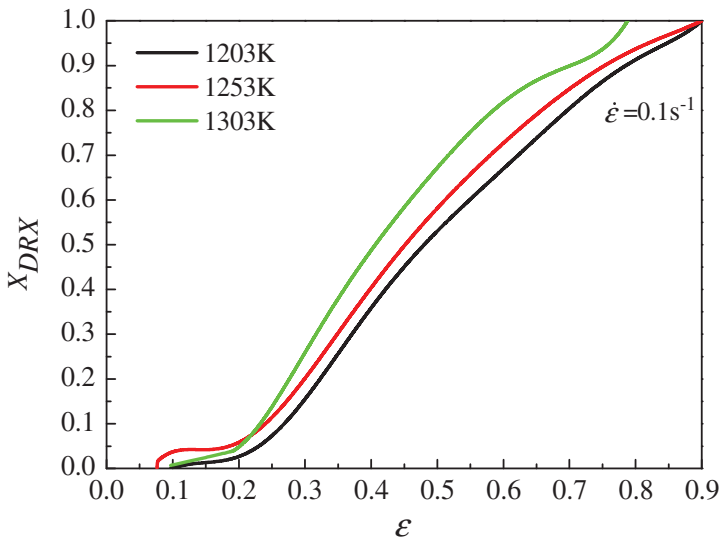

(b)

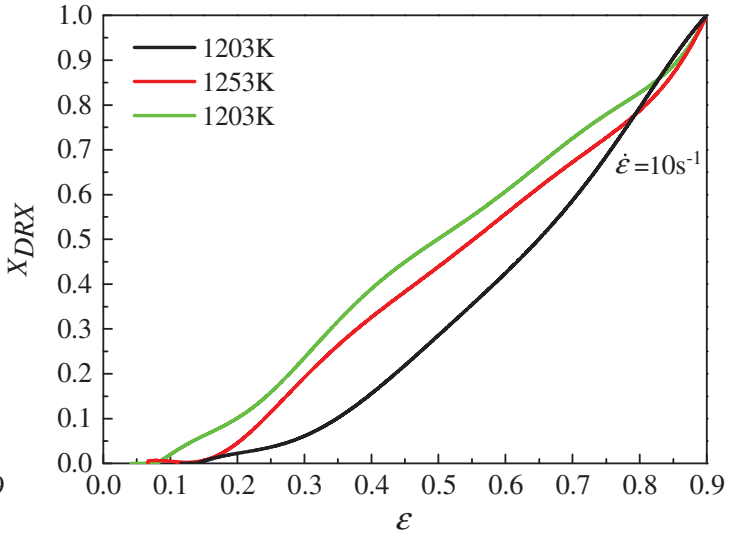

(d)

Figure 7: Predicted volume fractions of dynamic recrystallization obtained at different deformation temperatures and strain rates of: (a) $0.01 \mathrm{~s}^{-1}$, (b) $0.1 \mathrm{~s}^{-1}$, (c) $1 \mathrm{~s}^{-1}$, (d) $10 \mathrm{~s}^{-1}$. 


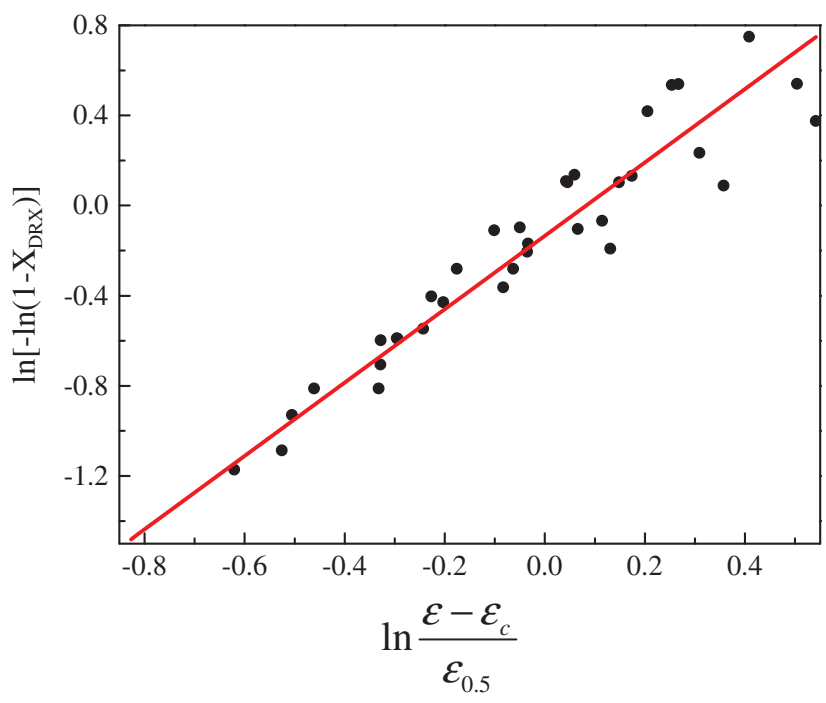

Figure 8: The relationships between $\ln \left[-\ln \left(1-X_{D R X}\right)\right]$ and $\ln \left[\left(\varepsilon-\varepsilon_{c}\right) / \varepsilon_{0.5}\right]$.

of $\ln \left[-\ln \left(1-X_{D R X}\right)\right]$ versus $\ln \left[\left(\varepsilon-\varepsilon_{c}\right) / \varepsilon_{0.5}\right]$ were obtained, which can be fitted linearly as shown in Figure 8 . Obviously, the value of the $k_{d}$ is the slope of this fitted line, and here its value was obtained as 1.6286. Then $k_{d}$ and a series of $X_{D R X}, \varepsilon_{0.5}, \varepsilon_{c}$ and $\varepsilon$ are substituted into eq. (8), and then the mean value of material constant $\beta_{d}$ was calculated as 0.9885 .

(2) Calculation of material constants: $m_{2}, \alpha_{2}, Q_{2}$

Taking natural logarithm on both sides of eqs (20), equation (20) can be represented as eq. (23).

$$
\ln \varepsilon_{0.5}=\ln \alpha_{2}+n_{2} \ln d_{0}+m_{2} \ln \dot{\varepsilon}+Q_{2} / R T
$$

Obviously, $m_{2}=\partial \ln \varepsilon_{0.5} / \partial \ln \dot{\varepsilon}$. As shown in Figure 9, through linear match, the relationships between $\ln \varepsilon_{0.5}$ and $\ln \dot{\varepsilon}$ under different temperatures were clear. It is

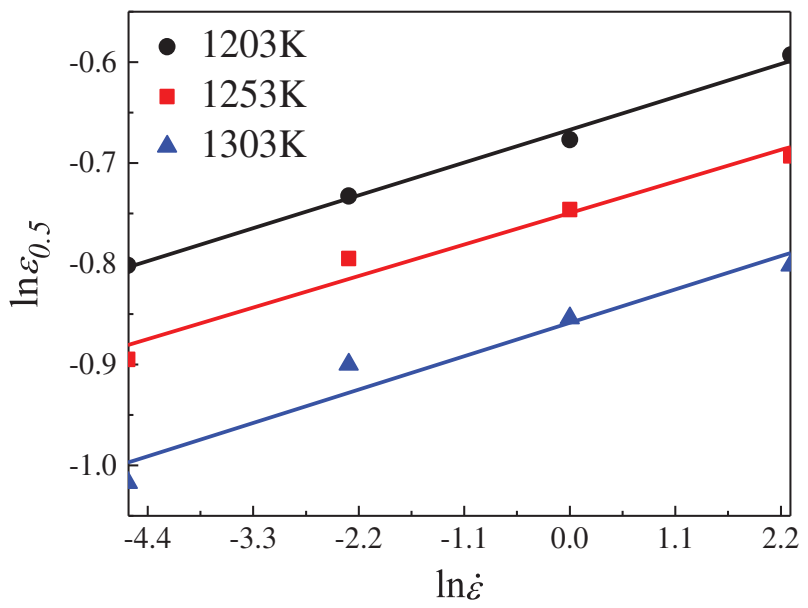

Figure 9: The relationships between $\ln \varepsilon_{0.5}$ and $\ln \dot{\varepsilon}$ found that the slope values of the three fitted lines were basic equal. Therefore, the value of the material constant, $m_{2}$, is recognized as the mean value of the three slope values, so its value was calculated as 0.0294 .

Observed from eq. (23), it is deduced that $Q_{2}=R \partial$ $\ln \varepsilon_{0.5} / \partial(1 / T)$. As shown in Figure 10, through linear match, the relationships between $\ln \varepsilon_{0.5}$ and $1 / \mathrm{T}$ under different strain rates appeared. It is found that the slope values of the four fitted lines were basic equal. Similarly, the value of the material constant, Q2, is recognized as the mean value of the four slope values, so its value was calculated as $36651 \mathrm{~J} \mathrm{~mol}^{-1}$.

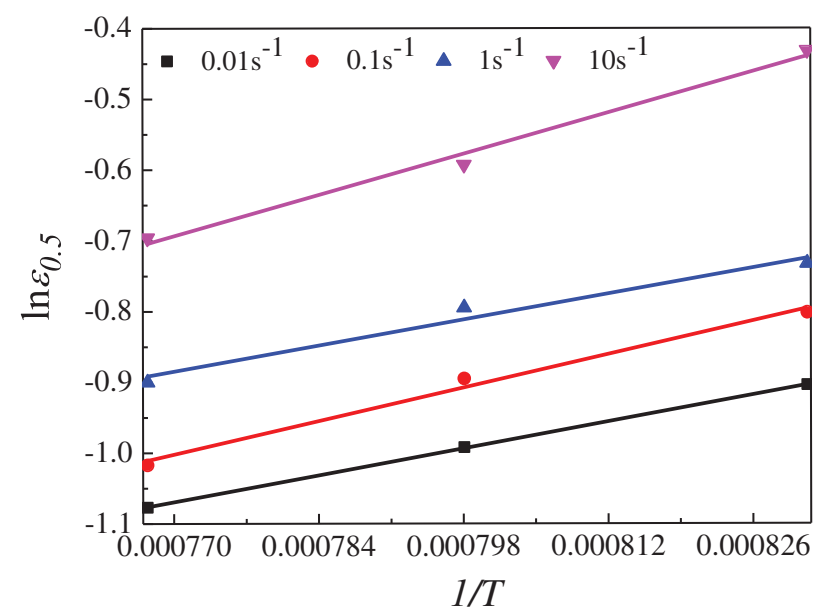

Figure 10: The relationships between $\ln \varepsilon_{0.5}$ and $1 / T$.

In present paper, the $n_{2}$ was set as 0 because that the effect of initial grain size is without consideration. Then take $m_{2}$ and $Q_{2}$ into eq. (23), twelve values of material constant, $\alpha_{2}$, under different deformation conditions can be calculated, and the value of $\alpha_{2}$ is accepted as the mean value of these twelve values, namely 0.0141 .

\section{Grain growth model}

The average austenite grain size of 3Cr20Ni10W2 under different temperature and strain rate has been published elsewhere [17], Based on these grain size, an empirical equation in Avrami type of the materials was utilized in this work, which is shown as following:

$$
d_{\text {drex }}=\alpha_{3} d_{0}{ }^{n_{3}} \dot{\varepsilon}^{m_{3}} \exp \left(Q_{3} / R T\right)
$$

where $R$ is the universal gas constant $\left(8.31 \mathrm{~J} \mathrm{~mol}^{-1} \mathrm{~K}^{-1}\right)$, $a_{3}, n_{3}$ and $m_{3}$ are material constants. In present work, the influence of different strain rate and temperature on the grain size was mainly investigated, so the eq. (24) can be simplified to eq. (25). 


$$
d_{\text {drex }}=\alpha_{3} \dot{\varepsilon}^{m_{3}} \exp \left(Q_{3} / R T\right)
$$

(1) Calculation of material constant $m_{3}, Q_{3}, \alpha_{3}$

Take natural logarithms on both sides of eq. (25), the eq. (26) is obtained.

$$
\ln d_{d r e x}=\ln \alpha_{3}+m_{3} \ln \dot{\varepsilon}+\left(Q_{3} / R T\right)
$$

The calculation method similar to the calculation for $m_{1}$, $Q_{1}, \alpha_{1}$ was conducted to determine the values of $m_{3}, Q_{3}$, $\alpha_{3}$. The values are $-0.0365,-36252.9816 \mathrm{~J} \mathrm{~mol}^{-1}$ and 750.892 respectively.

\section{Results and discussion}

\section{Microstructural evolution of DRX by CA simulation}

Figure $11(\mathrm{a} \sim \mathrm{g})$ illustrates the grain size evolution of the 3Cr20Ni10W2 sample at the temperature of $1203 \mathrm{~K}$ and at the strain rate of $0.01 \mathrm{~s}^{-1}$ by means of the CA simulation. It is observed from Figure 11 (a) that the recrystallization fraction increases with the increased simulation time. Moreover, Figure 11 shows that the mean grain size decreases gradually with the increased simulation time. Therefore, it is concluded that the grain size becomes smaller due to the DRX during the hot compression process. In addition, the recrystallized grains firstly occurred at the grain boundaries in the initial microstructure when the DRX starts, subsequently, these new grains grow up with the increase of deformation.
Meanwhile, the new crystal nuclei occur at the grain boundaries of the recrystallized grains formed previously as shown in Figure 11 (e), in other words, it can be summarized that the new recrystallized grains arise repeatedly until the plastic deformation ends as shown in Figure $11(\mathrm{~g})$, and DRX is precisely characterized by repeated nucleation and finite growth of the recrystallized grains.

\section{Prediction of grain size by CA simulation}

It is well know that the deformation temperatures and the strain rates have an important influence on the mean grain size during DRX. Based on CA simulation results, the effect of the deformation temperatures and the strain rates on the mean grain size is obtained. As shown in Figures 12 and 13, it is concluded that the mean grain size increases with the increased deformation temperature at a fixed strain rate, but decreases with the increased strain rate at a fixed temperature. This is because that both the decrease of deformation temperature and the increase of strain rate can cause the increase in the dislocation density, which contributes to accelerating DRX. Therefore, the recrystallized grains take place more repeatedly in the same deformation time.

\section{The comparison between experimental results and simulation results}

The error between the experimental grain size and the simulated grain size at different deformation conditions is

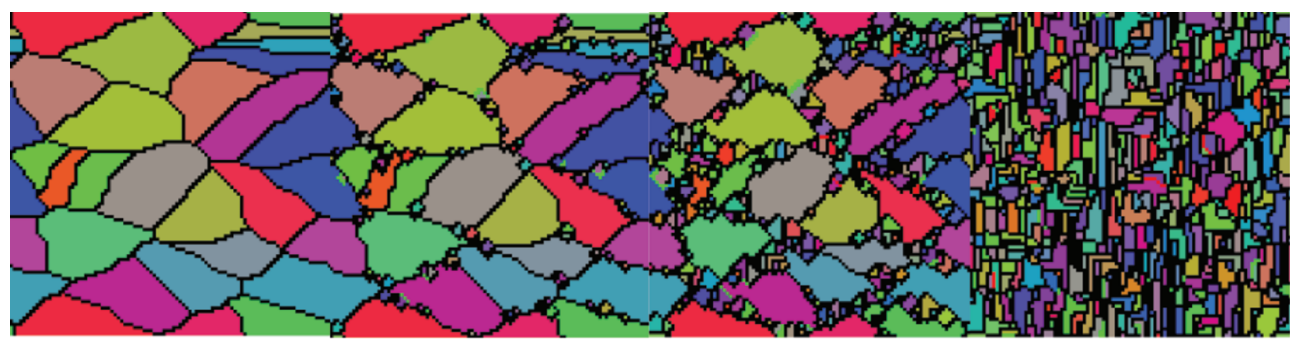

(a) (c)

(d)

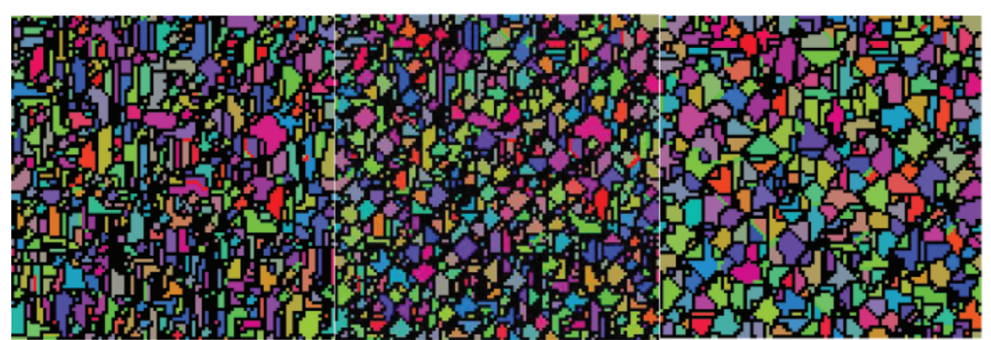

(e) (f) (g)
Figure 11: Microstructure evolution of the 3Cr20Ni10W2 at the deformation temperature of $1203 \mathrm{~K}$ and strain rate of $0.01 \mathrm{~s}^{-1}$. 

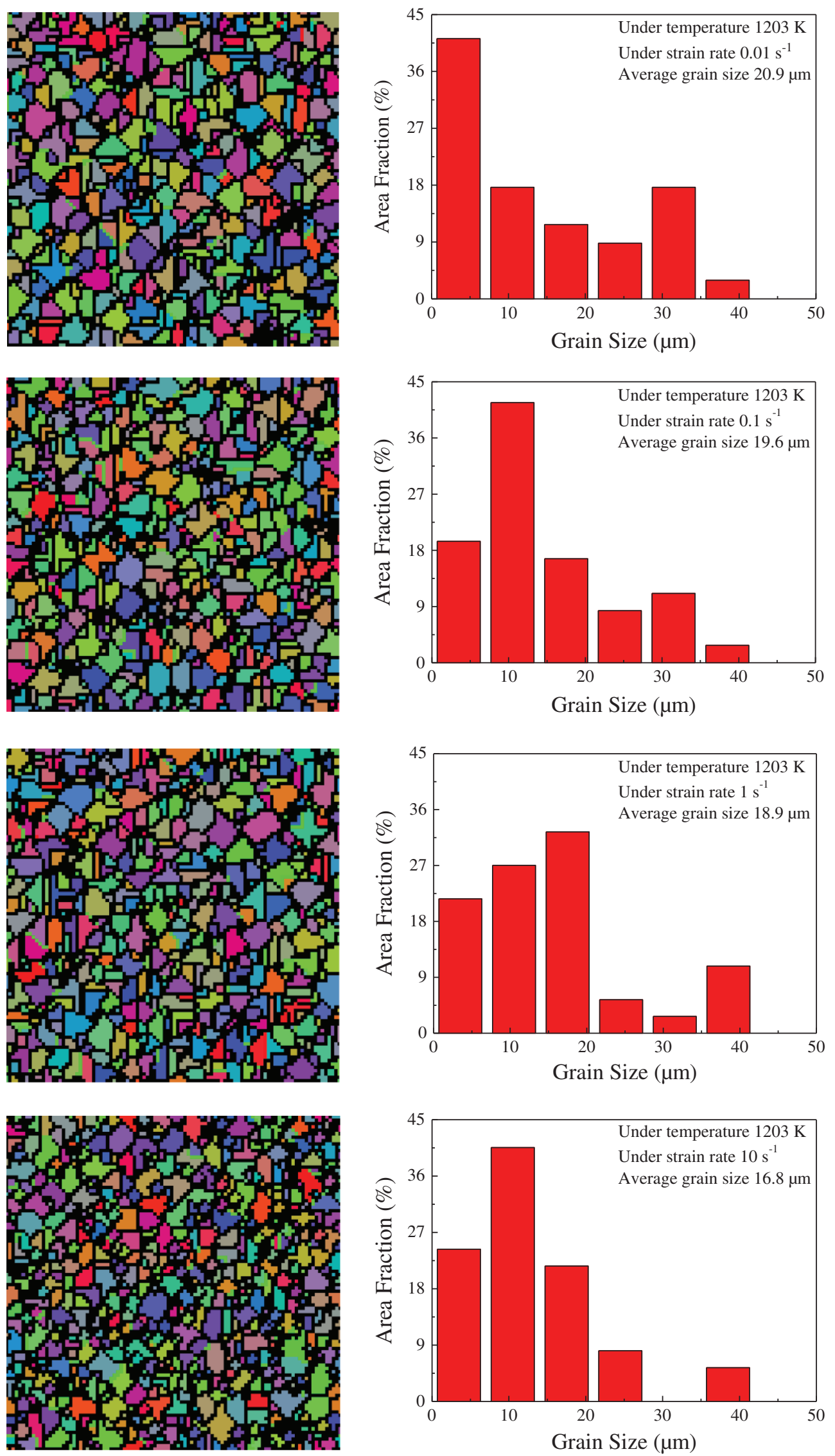

Figure 12: Prediction of microstructure and the simulated grain size at the deformation temperature of $1203 \mathrm{~K}$ and strain rates of: (a) $0.01 \mathrm{~s}^{-1}$ (b) $0.1 \mathrm{~s}^{-1}$ (c) $1 \mathrm{~s}^{-1}$ (d) $10 \mathrm{~s}^{-1}$. 

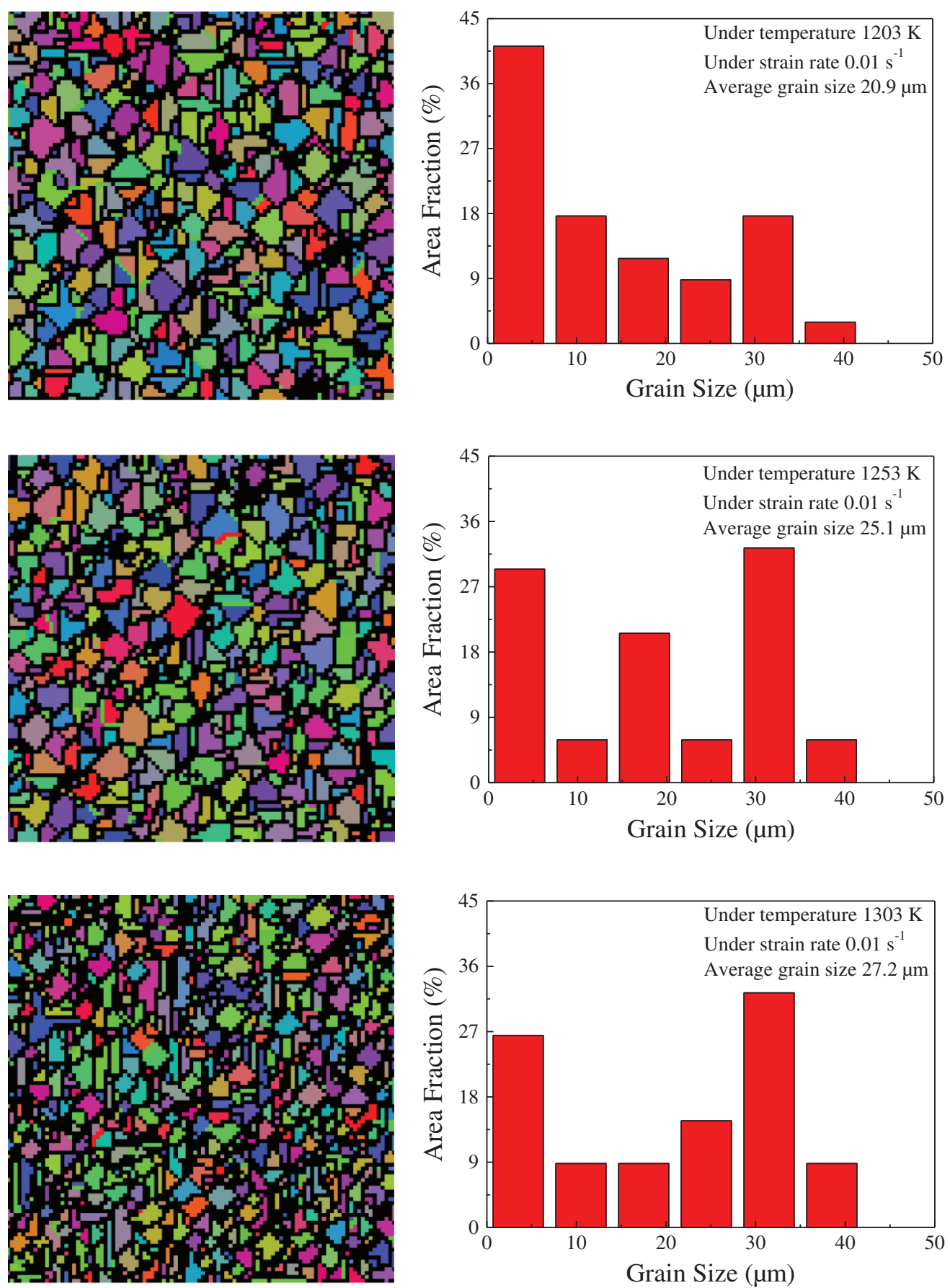

Figure 13: Prediction of microstructure and the simulated grain size at the fixed strain rate of $0.01 \mathrm{~s}^{-1}$ and deformation temperatures of: (a) $1203 \mathrm{~K}$ (b) $1253 \mathrm{~K}$ (c) $1303 \mathrm{~K}$.

elaborated by Table 1. It can be concluded that the grain size increases with the increased temperature under the same strain rate and the grain size decreases with the increased strain rate under the fixed temperature, which is consistent with the experimental results. In addition, the relative error between the experimental grain size and the simulated grain size at the deformation temperature of $1203 \mathrm{~K}$ and the strain rate of $0.01 \mathrm{~s}^{-1}$ reaches the minimum, which is $6.69 \%$. The maximum relative error is $16.01 \%$ at the deformation temperature of $1303 \mathrm{~K}$ and strain rate of $1 \mathrm{~s}^{-1}$. The average absolute relative error is $13.14 \%$, from which it can be summarized that the present CA model an efficient and proper method which can simulate the DRX behavior and calculate the final grain size accurately. 
Table 1: Relative error between experimental grain size and simulated grain size.

\begin{tabular}{lrrrr}
\hline $\begin{array}{l}\text { Strain } \\
\text { rate/s } \mathbf{s}^{-1}\end{array}$ & $\begin{array}{r}\text { Temperature } \\
\text { /K }\end{array}$ & Experimental & Simulated & $\begin{array}{r}\text { Relative } \\
\text { error/\% }\end{array}$ \\
\hline 0.01 & 1203 & 22.4 & 20.9 & 6.69 \\
& 1253 & 27.9 & 25.1 & 10.03 \\
& 1303 & 30.5 & 27.2 & 10.81 \\
0.1 & 1203 & 21.5 & 19.6 & 8.84 \\
& 1253 & 26.4 & 23.8 & 9.84 \\
& 1303 & 28.3 & 25.2 & 10.95 \\
1 & 1203 & 20.7 & 18.9 & 8.70 \\
& 1253 & 23.3 & 20.7 & 11.16 \\
& 1303 & 28.1 & 23.6 & 16.01 \\
10 & 1203 & 17.9 & 16.3 & 8.94 \\
& 1253 & 21.8 & 18.4 & 15.60 \\
& 1303 & 22.3 & 19.2 & 13.90 \\
& & & AARE (\%) 13.14 \\
\hline
\end{tabular}

\section{Conclusions}

Based on the analysis about the hot isothermal compressions strain-stress curves of the for as-extruded 3Cr20Ni10W2, the experimental data obtained from hot compression tests in the temperature range of 1203-1303 K with an interval of $50 \mathrm{~K}$ and strain rate range of $0.01-10 \mathrm{~s}^{-1}$ is adopted. After the establishment of the CA model, the DRX behavior of the 3Cr20Ni10W2 was studied, meanwhile the absolute average relative error indicates that the present CA model is able to investigate the microstructure evolution and calculate the grain size accurately during hot compression of 3Cr20Ni10W21 alloy. The main conclusions are summarized as following:

(1) Analysis on the hot isothermal compressions strainstress curves of the 3Cr20Ni10W2 indicates that DRX occurred under the deformation conditions including 1203 1303 $\mathrm{K} \& 0.01 \mathrm{~s}^{-1}, 1203 \sim 1303 \mathrm{~K} \& 0.1 \mathrm{~s}^{-1}$, $1203 \sim 1353 \mathrm{~K} \& 1 \mathrm{~s}^{-1}$ and $1203 \sim 1403 \mathrm{~K} \& 10 \mathrm{~s}^{-1}$. Therefore, the temperature range of 1203-1303 K with an interval of $50 \mathrm{~K}$ and strain rate range of 0.01-10 s ${ }^{-1}$ is adopted to establish the CA model.

(2) Dislocation density model, recovery model, the DRX nucleation and growth model and DRX volume fraction model and grain size model are established successively. During this process, the value of different coefficient was determined, which lay a foundation for the subsequent CA model.

(3) The simulated results shows that the recrystallized grains firstly occur at the grain boundaries of the grains in the initial microstructure when the DRX starts, subsequently, these new grains grow up with the increase of deformation. In addition, the mean grain size increases with the increased deformation temperature at a fixed strain rate, but decreases with the increased strain rate at a fixed temperature. The average absolute relative error also indicates that the present CA model is able to simulate the DRX behavior and calculate the final grain size accurately.

Acknowledgments: This work was completed with the support of Talented young talents project (No. 2014000020124G096) and the Beijing finance found of young prominent talent projects (No. 2014000026833ZK25).

\section{References}

[1] R. Damodaram, S.G.S. Raman and K.P. Rao, Mater. Sci. Eng., 560 (6) (2013) 781-786.

[2] H.S. Jeong, J.R. Cho, N.K. Lee and H.C. Park, Mater. Sci. Forum, 510 (2006) 142-145.

[3] K. Shelton and D.C. Dunand, Acta Mater., 44 (11) (1996) 4571-4585.

[4] W. Roberts and B. Ahlblom, Acta Metallurgica, 26 (5) (1978) 801-813.

[5] Y. He, H. Ding, L. Liu and K. Shin, Mater. Sci. Eng., 429 (1-2) (2006) 236-246.

[6] P. Peczak and M.J. Luton, Acta Metallurgica Et Materialia, 41 (1) (1993) 59-71.

[7] K.G.F. Janssens, Math. Comput. Simul., 80 (7) (2010) 1361-1381.

[8] Q. Du, D.Z. Li and Y.Y. Li, Journal of Materials Science \& Technology, 16 (6) (2000) 568-572.

[9] H. Wang and E.H. Han, J. Mater. Sci. Technol., 28 (5) (2012) 427-432.

[10] L. Li, F. He, X. Liu, Y. Lou, J. Zhou and J. Duczczyk, Key Eng. Mater., 491 (2011) 265-272.

[11] S. Gourdet and F. Montheillet, Acta Mater., 51 (9) (2003) 2685-2699.

[12] F. Chen, Z. Cui, J. Liu, X. Zhang and W. Chen, Modelling Simulation Mater. Sci. Eng., 17 (7) (2009) 075015.

[13] Z. Jin and Z. Cui, Mater. Sci. Eng., 527 (13-14) (2010) 3111-3119.

[14] E. Afshari and S. Serajzadeh, J. Mater. Eng. Perfor., 21 (8) (2012) 1553-1561.

[15] Y.X. Liu, Y.C. Lin, H.B. Li, D.X. Wen, X.M. Chen and M.S. Chen, Mater. Sci. Eng., 626 (2015) 432-440.

[16] Y.F. Xia, G.C. Luo, D.S. Wu, G.Z. Quan and J. Zhou, Adv. Mechanical Eng., 5 (3) (2015) 520127-520127.

[17] G.Z. Quan, A. Mao, Z.Y. Zou, G.C. Luo and J.T. Liang, High Temp. Mater. Proc., 34 (7) (2015) 697-713.

[18] Y.X. Liu, Y.C. Lin and Y. Zhou, Mater. Sci. Eng., 691 (2017) 88-99.

[19] Y.C. Lin, Y.X. Liu, M.S. Chen, M.H. Huang, X. Ma and Z.L. Long, Mater. Des., 99 (2016) 107-114.

[20] A. Laasraoui and J.J. Jonas, Metallurgical Mater. Trans., 22 (7) (1991) 1545-1558. 
[21] R.C. Souza, E.S. Silva, A.M. Jorge, J.M. Cabrera and O. Balancin, Mater. Sci. Eng., 582 (2) (2013) 96-107.

[22] L.A. Reyes, P. Páramo, A.S. Zamarripa, M.D.L. Garza and M.P.G. Mata, Mater. Des., 83 (2015) 301-307.

[23] R.L. Goetz and V. Seetharaman, Metallurgical Mater. Trans., 29 (9) (1998) 2307-2321.

[24] S. Mandal, S.K. Mishra, A. Kumar, I. Samajdar, P.V. Sivaprasad, T. Jayakumar and B. Raj, Philosophical Magazine, 88 (6) (2008) 883-897.

[25] R. Ding and Z.X. Guo, Acta Mater., 49 (16) (2001) 3163-3175.

[26] H. Hallberg, M. Wallin and M. Ristinmaa, Computational Mater. Sci., 49 (1) (2010) 25-34.

[27] W.T. Read and W. Shockley, Phys. Rev., 78 (3) (1950) 275-289.
[28] Z. Yang, Y.C. Guo, J.P. Li, F. He, F. Xia and M.X. Liang, Mater. Sci. Eng., 485 (1-2) (2008) 487-491.

[29] K.L. Wang, M.W. Fu, S.Q. Lu and X. Li, Mater. Des., 32 (3) (2011) 1283-1291.

[30] G. Ji, F. Li, Q. Li, H. Li and Z. Li, Mater. Sci. Eng., 527 (9) (2010) 2350-2355.

[31] Y.C. Lin, M.S. Chen and J. Zhong, Mater. Des., 30 (3) (2009) 908-913.

[32] S.H. Zahiri, C.H.J. Davies and P.D. Hodgson, Scr Mater., 52 (4) (2005) 299-304.

[33] Y.C. Lin and X.M. Chen, Mater. Des., 32 (4) (2011) 1733-1759.

[34] J.J. Jonas, X. Quelennec, L. Jiang and É. Martin, Acta Mater., 57 (9) (2009) 2748-2756. 